\title{
IN VITRO EFFECTS OF CAFFEINE ON THE PROLIFERATION, APOPTOSIS AND GENE TRANSCRIPTS EXPRESSION OF CHONDROGENIC DIFFERENTIATION IN GROWTH CARTILAGE OF RATS
}

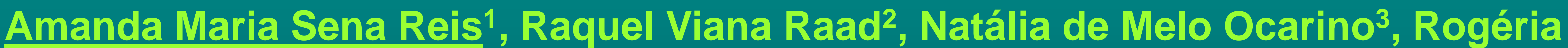 Serakides ${ }^{3}$}

1 Mestranda, Escola de Veterinária, Universidade Federal de Minas Gerais, Belo Horizonte, Minas Gerais, Brasil.

2 Aluna de Iniciação científica, Escola de Veterinária, Universidade Federal de Minas Gerais, Belo Horizonte, Minas Gerais, Brasil. 3 Professora, Escola de Veterinária, Universidade Federal de Minas Gerais, Belo Horizonte, Minas Gerais, Brasil (E-mail: serakidesufmg@gmail.com).

\section{INTRODUCTION}

Caffeine is a methylxanthine found in several foods, therefore widely consumed by humans. A variety of its effects and mechanisms on tissues have been widely studied, however, despite the fact that it alters post-natal bone growth, there have been only a few studies on its effects on growth cartilage. The objective of this study is to evaluate the in vitro effects of caffeine on proliferation, apoptosis and gene transcripts expression of chondrogenic differentiation in growth cartilage.

\section{MATERIAL AND METHODS}

The cartilaginous epiphyses of 80 femurs of newborn rats, which were divided into two subgroups: treated with caffeine and control group, both observed the time of $0,7,14$ and 21 days. The cartilaginous epiphyses of four femurs of each subgroup and each time span were subjected to histomorphometric and immunohistochemical analysis and Tunel in order to evaluate cell proliferation and apoptosis, respectively. The cartilaginous epiphyses of six femurs were subjected to RT-PCR in real time to evaluate the expression of caspase-3, Runx-2 and Sox-9.

\section{RESULTS AND DISCUSSION}

Both the control group and the group treated with caffeine, chondroblasts morphology was similar throughout the period of cultivation. The percentage of empty lacunae in the chondroblasts of the cartilaginous epiphysis of the femur was increased significantly throughout the cultivation period. However, after 21 days the group treated with caffeine showed the number of empty lacunae of chondroblasts significantly lower compared to the control group (Figure 1). The decrease of proliferative activity and the increase of chondroblasts in apoptosis up to 21 days were found regardless of the subgroup. However, the decrease in cell proliferation caused by caffeine was significantly lower compared to the control group (Figure 2) and significantly increased the expression of gene transcripts for chondrogenic differentiation, represented by Sox-9 and the Runx-2 (Figure 4). However, the in vitro culture with caffeine revealed antagonistic effects: despite the positive effect on the proliferation and differentiation of chondroblasts, caffeine increased apoptosis, characterized by increased expression of caspase 3 (Figure 4 ) and of the number of cells undergoing apoptosis ( Figure 3 ), $(p<0.05)$.

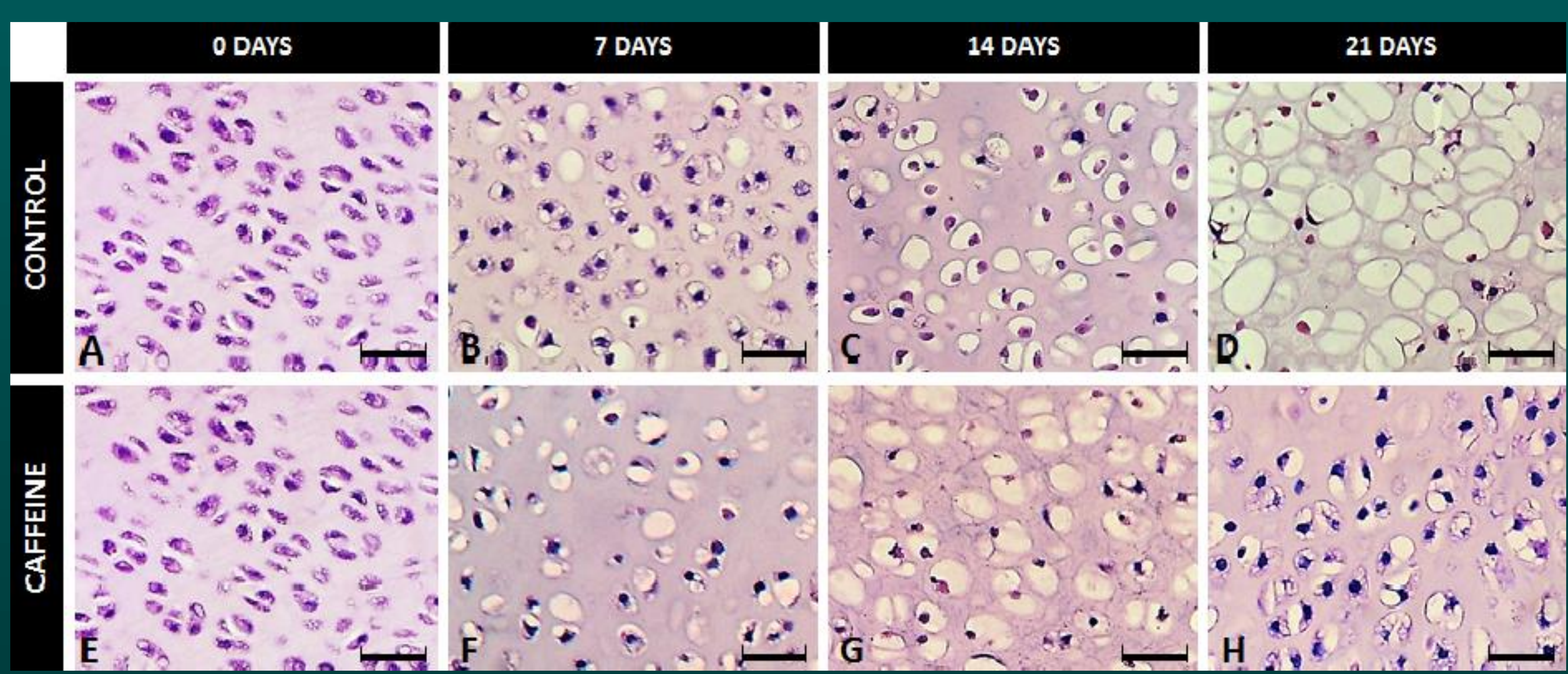

Figure 1: Cartilaginous epiphysis of newborn rats cultivated in culture with or without caffeine (control). $\mathrm{HE}$, Bar= with the number of empty lacunae of chondroblasts during the entire period of culture in comparison to day 0 . At 21 days of culture, group treated with caffeine presented a lower number of empty chondroblasts lacunae in comparis to
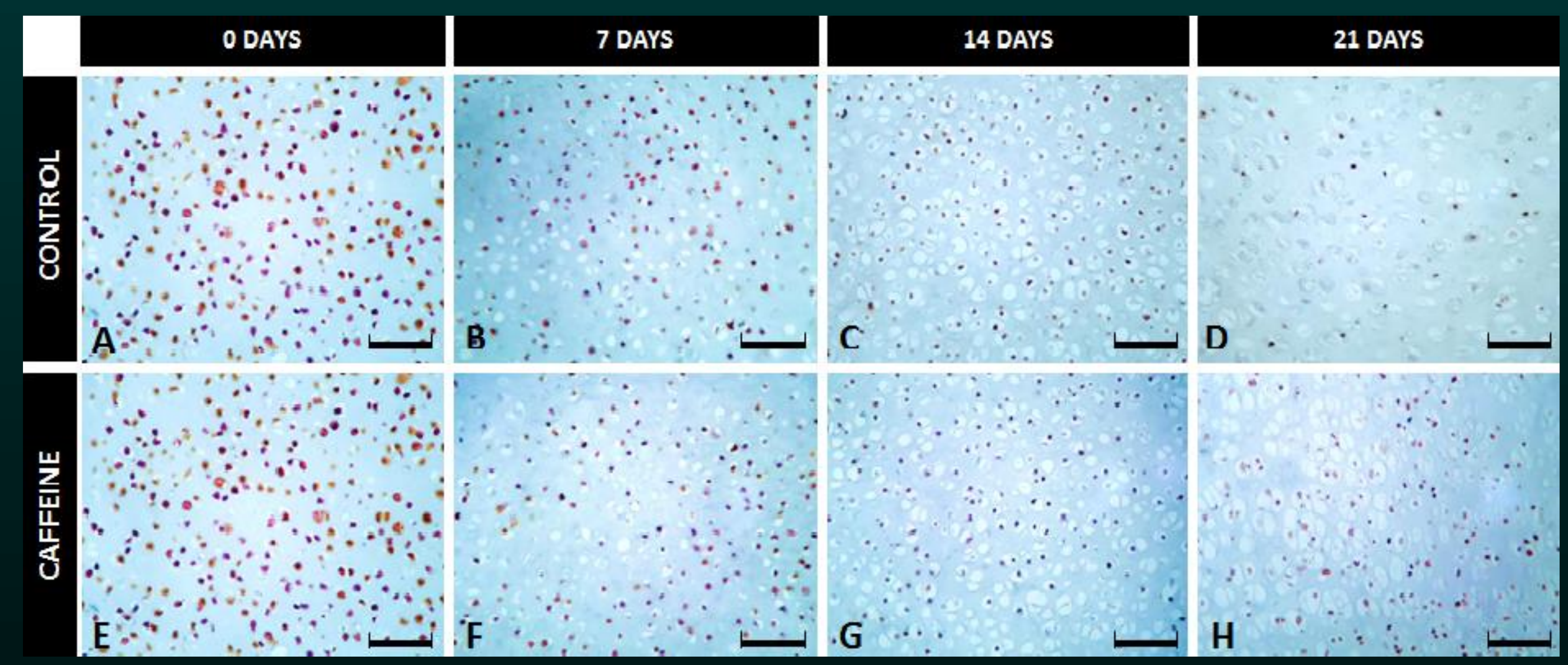

Figure 2: Cartilaginous epiphysis of newborn rats cultivated in culture with or without caffeine (control).

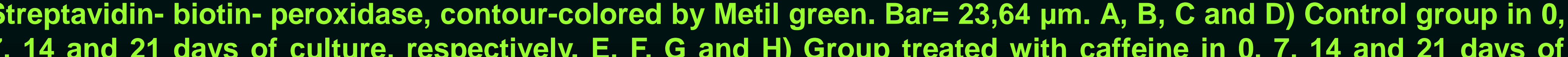

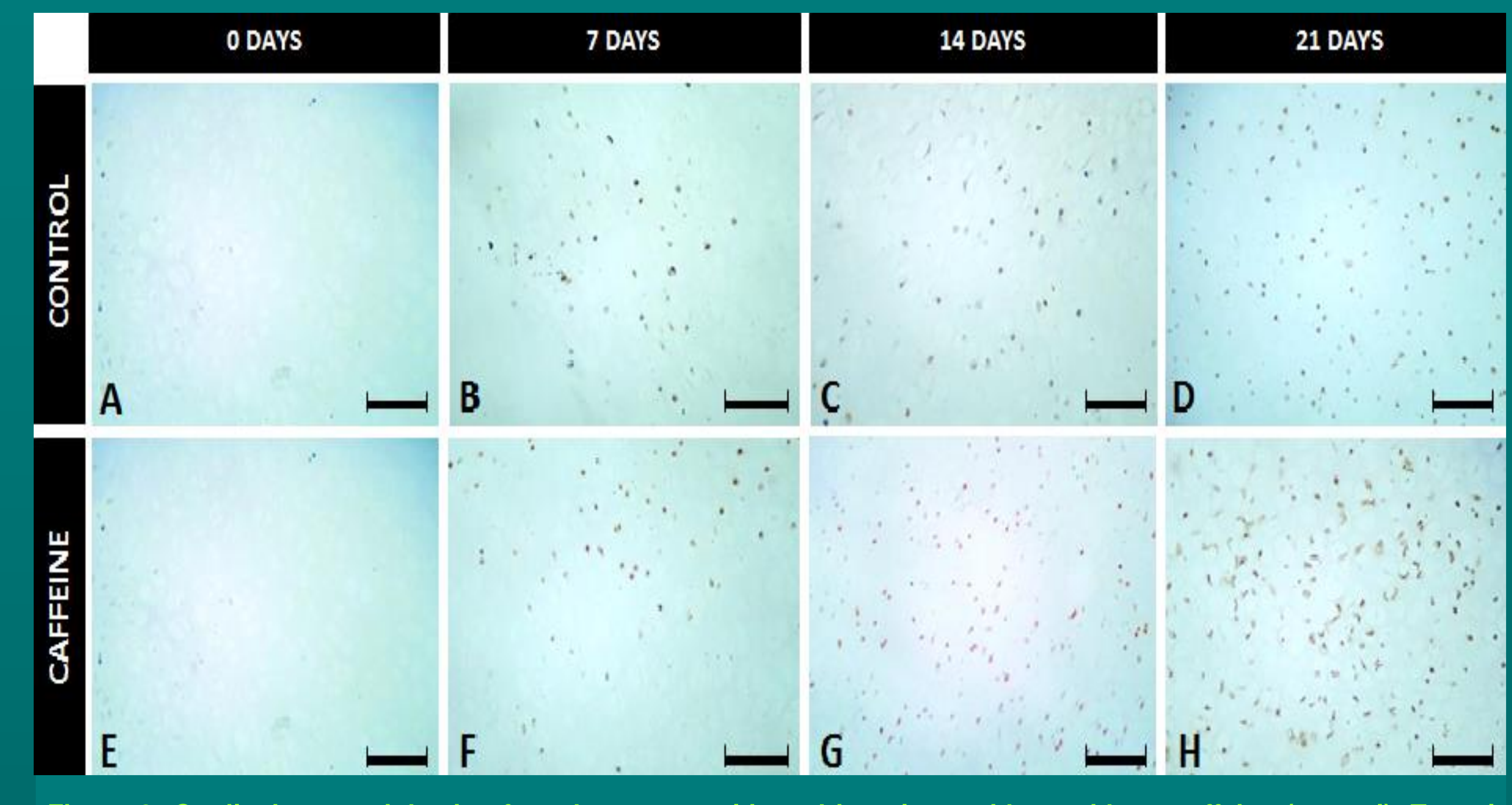

Figure 3: Cartilaginous epiphysis of newborn rats cultivated in culture with or without caffeine (control). Tunnel
technique, contour- colored by Metil green. Bar= 23,64 um. A, B, C and D) Control group in $0,7,14$ and 21 days of culture respectively. E, F, G and H) Group treated with caffeine in $0,7,14$ and 21 days of cultive, respectively.
Control and treated groups gradual increase in the number of apoptotic chondroblasts, in brown, during the entire period of culture in comparison to day 0 . At 21 days of culture, the group treated with caffeine presented higher number of apoptotic chondroblast in comparison to control group in the same period.

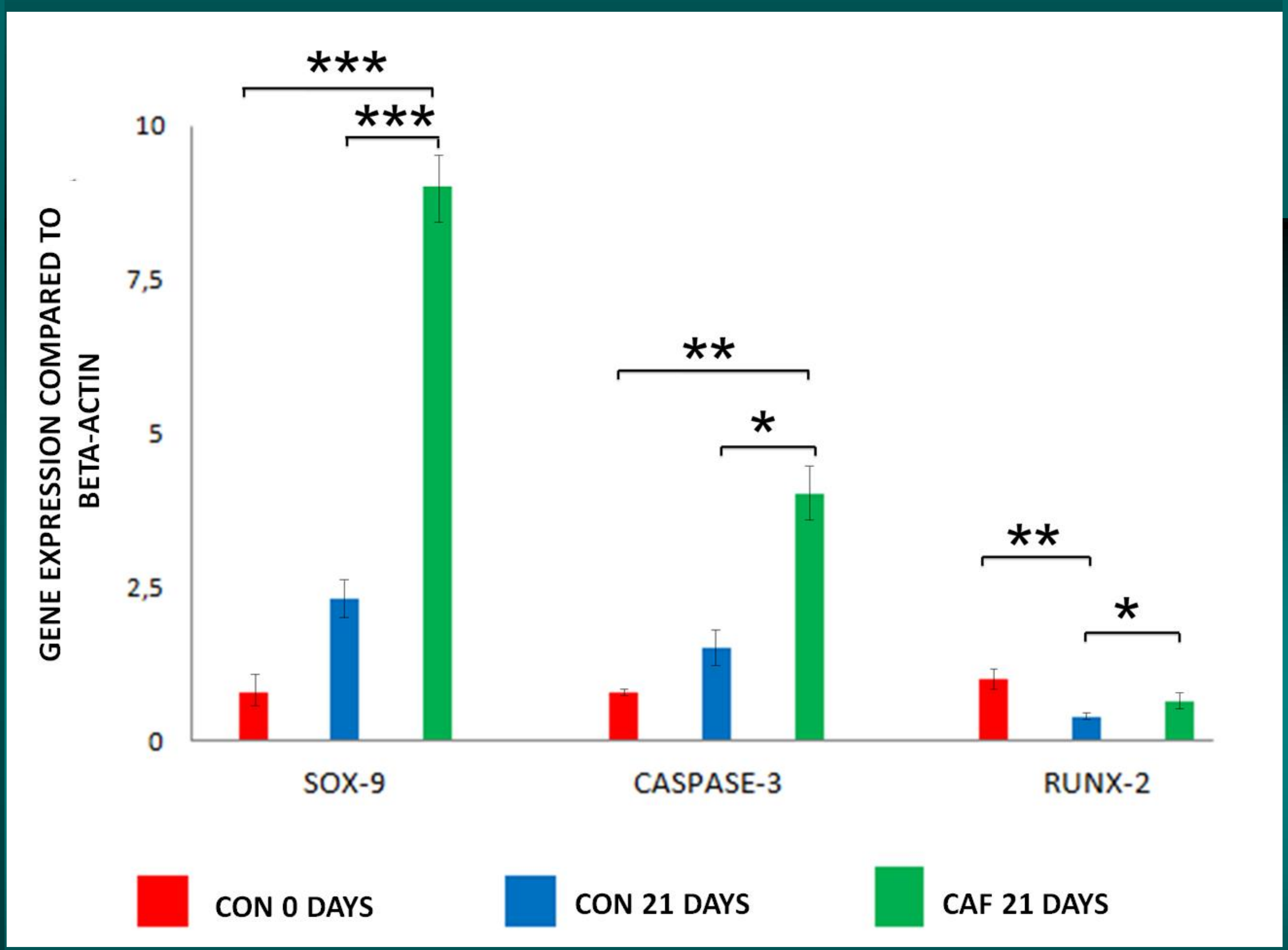

Figure 4: Graphic illustrating an increase in the gene transcripts caspase- 3 and Sox-9 from the cartilaginous epiphysis of the femurs of newborns from the treated group with caffeine at 21 days, by RT-PCR technique. The expression of Runx-2 was lower in control group at 21 days of culture, in comparison to day 0 . But on the group comparison to control group $\left({ }^{*} p<0,05\right)$.

\section{CONCLUSION}

It follows that the caffeine presents antagonistic effects in vitro on growth cartilage, increasing the proliferation, differentiation and cell apoptosis.

\section{REFERENCES}

WAKITA, R.; IZUMI, T.; ITOMAN, M.. Thyroid hormone-induced chondrocyte terminal differentiation in rat femur organ culture. Cell Tissue Res., v.293, p 357-364, 1998. 